\title{
Solar Observation Target Identification Convention for use in Solar Physics
}

Published online: 20 April 2010

(C) Springer Science+Business Media B.V. 2010

\section{Motivation:}

We strongly encourage the use of a standardized target identification to be included in publications on solar events. The primary purpose is to enable the automated identification of publications on the same event, or on other related events, in the on-line literature by search engines such as the Astrophysics Data System (ADS). The convention does not aim to categorize or classify events, but is limited specifically to the identification of regions in space and intervals in time within which events occur.

In the astrophysical literature, the inclusion of target names enables readers and search engines to find publications on the same target based on other observations or other analyses of the same observations. Even though targets are generally known by multiple names, multicatalog services such as SIMBAD readily enable the mapping from one name standard to another.

Solar physics has largely done without such a target naming convention. One exception is the use of NOAA active region numbers, but even that is not standardized in the literature. Consequently, finding studies on a particular event, or related studies on nearby events, is difficult, cannot be automated, and is often a matter of chance or serendipity. We encourage the use of a standardized convention for the identification of solar events in order to make (human and automated) searches for particular events easier, and to facilitate the development of autonomous search software in, e.g., ADS.

\section{Attributes:}

A solar-event naming convention should apply to all types of solar phenomena, be independent of the observatory perspective, and be as concise as possible so that minimal effort is needed for its use. In order for the names to be usable, they obviously need to contain time and location information. As solar events have an extent in both space and time, ranges may in principle be included, but we suggest that this is not necessary: the association of events close in time and space can be left to the user of the search engine who can specify the desired ranges. Moreover, as the primary purpose is to enable (autonomous) identification of publications on events analyzed by different groups or observed by different observatories, we argue that a precision to the nearest heliocentric degree and to the nearest second 
suffices. The primary purpose is to indicate a region in space-time, not to flag particular events; hence, no event specification is attached to the locator.

Consequently, the minimal form of a Solar Object Locator (SOL) contains only time (in UT, for events seen in, e.g., full-Sun light curves, such as the GOES flares, or for CMEs whose origin is unknown or ambiguous). The standard locator combines time with Carrington longitude and co-latitude (between 0 and 360 degrees and 0 and 180 degrees, respectively). A full locator could also contain a radial coordinate (in solar radii from Sun center).

Standard for the identification of solar events:

minimal: SOLyyyy-mm-dd

standard: SOLYYYY-mm-ddThh: $\mathrm{mm}:$ ssLdddCddd

allowed (for, e.g., long-lived active regions): SOLyyyy-mm-ddLdddCddd

full: SOLyYyy-mm-ddThh:mm: ssLdddCdddRnnn.f

(date and time in UT using the IAU approved FITS standard format; positive-definite Carrington longitude and co-latitude in degrees; radial distance from Sun center in solar radii). Ranges (including source extent or position uncertainties) could be added if desired.

The standard uses Carrington coordinates which enable, for example, inclusion of observations seen from any observatory, whether on Earth or not (e.g., STEREO). We welcome suggestions on how to extend this standard to heliospheric observations from different vantage points where precise longitude information may be unavailable.

\section{Implementation}

The introduction of this target naming convention on a voluntary basis in on-line versions of manuscripts is supported by the editors of Solar Physics, as well as by the Virtual Solar Observatory, and IAU Commissions 10 and 12. As a start, we envisage their use in keywords, text, and possibly as electronic supplementary material along with other meta-data such as sponsors and data providers. After a six-month period for comments, we are ready to adopt this convention.

John Leibacher, Takashi Sakurai, Karel Schrijver (Editorial Board Chair), and Lidia van Driel-Gesztelyi

1 January 2010 